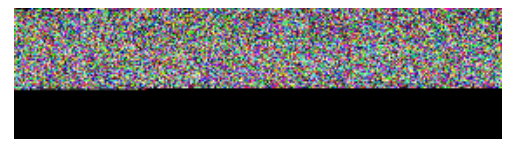

This information is current as of April 26, 2023.

\title{
Tumors of the Spine
}

AJNR Am J Neuroradiol 2009, 30 (9) E137 doi: https://doi.org/10.3174/ajnr.A1719 http://www.ajnr.org/content/30/9/E137 


\section{BOOK REVIEW}

\section{Tumors of the Spine}

D.H. Kim, U.-K. Chang, S.-H. Kim, M.H. Bilsky, eds. Saunders Elsevier; 2008, 728 pages, 600 illustrations, \$280.00.

T umors of the Spine, a multiauthored (22 authors) 728-page hardcover book edited by 4 spine neurosurgeons (Drs Kim, Chang, Kim, and Bilsky), is a densely illustrated and highly readable text, which should have appeal to neurosurgeons, orthopedic surgeons, and neuroradiologists alike. As is the increasing trend these days, the book comes with a CD, which contains images shown in the printed text. This feature adds immensely to the value of the textbook. What is curious, though, is that the opened CD is entitled "Tumors of the Spone" $[\mathrm{sic}]$. Someone clearly did not carefully proofread this part of the CD.

The book is rich with radiographic images (primarily MR images), pathologic specimens (particularly photomicrographs), intraoperative photographs, and excellent line drawings of surgical concepts and devices. The latter helps immensely in understanding surgical approaches, particularly in various stabilization procedures following tumor resection. With the various and sundry constructs now used, these chapters are particularly helpful to the radiologist, who is often confronted with an ever-changing plethora of prosthetic implantable stabilization devices.

The book is divided into 5 sections:

1) "Classification of Spine Tumors" ("Extradural," "Intradural Extramedullary," "Intramedullary").

2) "Evaluation of the Patient with Spinal Tumors" ("Neurologic," "Radiographic," "Electrodiagnostic," "Metastatic").

3) "Adjunct Therapy" ("Chemotherapy," "Endovascular Therapy," "Radiation Therapy").

4) "Surgical Treatment for Spinal Tumors" ("Percutaneous Biopsy," "Cervical Approach," "Thoracic Approach," "Lumbar Approach," "Intradural Tumors," "Minimally Invasive Techniques").

5) "Spine Stabilization" ("Craniovertebral and Cervical Junction," "Thoracic Spine," "Lumbar Spine”).
As one reads through the opening chapters, some questions arise relative to the images presented. A case of an osteochondroma is described as originating from the transverse process of the thoracic spine; however, the only abnormality visible on the images is high attenuation surrounding the posterior elements of $\mathrm{C} 1$. In general, chapters written by authors

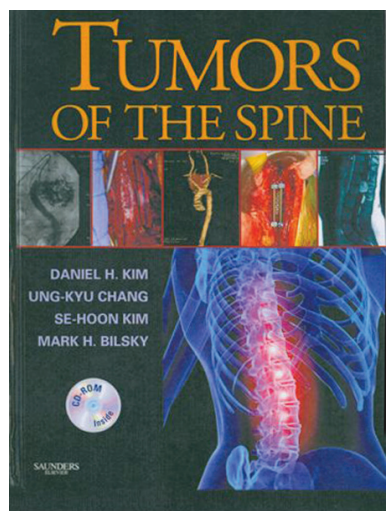
other than the 2 neuroradiologists (Drs Lane and Do) have many images that are not described; and in many instances, there is crucial information missing from the legends and/or the discussion (eg, canal invasion by bony or soft-tissue masses) or there is a lack of differential considerations in the discussion. Some legends do not have the diagnosis, and one has to look back through the text to see what diagnosis pertains to that particular image. Many of the images in the early chapters are poorly windowed, poorly centered to the area of interest, and lack labeling to direct the reader with minimum experience in interpretation to the area of interest. Most amazing, the authors, editors, and publisher even neglected to crop out the patient's name in a case of fibrous dysplasia of the spine. The conclusion reached by this reviewer was that some of the chapters were poorly scrutinized for content and image acceptability. Also, some terminology is wanting-such as calling an "intradural meningioma" a "spinal cord meningioma."

Despite these reservations relative to the radiology, the true value of the book to our specialty is to develop an understanding of the surgical approach and management of a wide range of spinal tumors. This book can be confidently recommended to the libraries of departments and sections of neuroradiology. It can serve as a resource when questions arise relative to imaging and the operative/postoperative evaluation of patients with spine tumors.

DOI 10.3174/ajnr.A1719 